\title{
An Extension to the Navier-Stokes Equations to Incorporate Gas Molecular Collisions with Boundaries
}

\author{
Erik J. Arlemark* S. Kokou Dadzie \\ Jason M. Reese \\ Department of Mechanical Engineering, \\ University of Strathclyde, \\ Glasgow G1 1XJ, \\ United Kingdom
}

\begin{abstract}
We investigate a model for micro-gas-flows consisting of the Navier-Stokes equations extended to include a description of molecular collisions with solid boundaries, together with first and second order velocity slip boundary conditions. By considering molecular collisions affected by boundaries in gas flows we capture some of the near-wall effects that the conventional Navier-Stokes equations with a linear stress/strain-rate relationship are unable to describe. Our model is expressed through a geometry-dependent mean-free-path yielding a new viscosity expression, which makes the stress/strain-rate constitutive relationship non-linear. Test cases consisting of Couette and Poiseuille flows are solved using these extended Navier-Stokes equations, and we compare the resulting velocity profiles with conventional Navier-Stokes solutions and those from the BGK kinetic model. The Poiseuille mass flow-rate results are compared with results from the BGK-model and experimental data, for various degrees of rarefaction. We assess the range of applicability of our model and show that it can extend the applicability
\end{abstract}

\footnotetext{
${ }^{*}$ Corresponding author: e-mail: erik.arlemark@strath.ac.uk
} 
of conventional fluid dynamic techniques into the early continuum-transition regime.

We also discuss the limitations of our model due to its various physical assumptions, and we outline ideas for further development.

keywords: Micro Gas Flows, Navier Stokes Equations, Mean Free Path, Non Linear Constitutive Relationships, Velocity Slip, Knudsen Layer

\section{List of Figures}

1 A molecule confined between two planar walls with spacing $H \ldots \ldots$

2 A molecule at a distance $H / 2+y$ from a planar wall. . . . . . . . . . . . 11

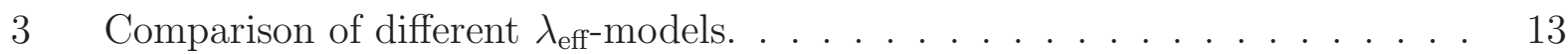

4 Couette flow velocity profiles for $K n 0.01,0.04$ and $0.08 \ldots \ldots \ldots$

$5 \quad$ Couette flow velocity profiles for $K n 0.01,0.113$ and $0.339 \ldots \ldots \ldots$

$6 \quad$ Poiseuille flow velocity profiles for $K n$ 0.01, 0.04 and 0.08 . . . . . . . . 20

$7 \quad$ Poiseuille flow velocity profiles for $K n$ 0.113, 0.339 and $0.903 \ldots \ldots \ldots$

8 Mass flow results. . . . . . . . . . . . . . . . . . . . . . . 24

\section{List of Tables}

1 Applicability ranges of the tested NS-based models. . . . . . . . . . . . . . 25 


\section{Introduction}

As interest in micro-gas-flow applications is growing with improved manufacturing capabilities, it is also becoming more widely appreciated that the conventional incompressible Navier-Stokes (NS) equations with no-velocity-slip boundary conditions fail to predict many of these flows properly. This is because micro-gas-flows differ from macro-gas-flows due to the relatively large ratio of the confining boundary surface area to the volume of the confined gas, meaning that certain surface effects must be taken into account. These surface effects considerably influence the flow in the near-wall region (the Knudsen-layer) which, because of the small scale of the system, comprises a substantial volume of many micro-gas-flows. The width of this Knudsen-layer is usually expressed in terms of the average travelling distance of molecules between intermolecular collisions - the mean free path, $\lambda$. The Knudsen-layer is about one to two mean free paths wide.

To indicate the degree of rarefaction, or state of non-equilibrium, of gas flows the key parameter is the Knudsen number,

$$
K n=\frac{\lambda}{H},
$$

where for example, for a micro-channel the full height, $H$, of the channel is the measure of the system length scale. Micro-gas-flows often have relatively large $K n$ due to the small length scales, and certain rarefaction effects then become apparent. Experiments, such as those performed by Arkilic et al. [1] and Colin [2], have shown that the NS equations cannot capture the correct mass flow rates along a micro-channel without requiring a velocity slip boundary condition to be applied. The conventional no-slip boundary condition commonly used with NS is valid only for cases where the gas is in a state of near-equilibrium. Micro-gas- 
flows of relatively large $K n$ require different boundary conditions [3]. The lower threshold value for applying a slip boundary condition is generally $K n=0.01$, which therefore is referred to as the lower limit of the "slip regime". For gas-flows at larger $K n$, further modelling modifications are needed because the linear constitutive NS relationships break down. This happens at about $K n=0.1$, which is known as the lower limit of the "continuumtransition" regime: the gas-flow is not modelled well either by a conventional continuum description nor by a free molecular description. This is the regime that we mostly focus on in this paper.

Modelling of surface effects in the Knudsen-layer should ideally be performed using kinetic theory. However an approximate extension to the NS model would be less demanding in terms of computational capacity, and the simplicity and practicality of NS make it desirable to solve flow cases using this model for as high- $K n$ flows as possible. As suggested by Guo et al. [4] NS may be extended to larger $K n$ by incorporating the gas molecular interactions with unyielding boundary walls through modification of the conventional expression of the molecular mean free path. In this paper, by considering the molecular interactions with the walls, we obtain an "effective" geometry-dependent mean free path, $\lambda_{\text {eff }}$, which is in turn used to obtain an "effective" fluid viscosity, which follows through into a non-linear stress/strain-rate relationship.

We solve our new NS model for isothermal cases using velocity slip boundary conditions of first order as well as of second order and focus on the predictions of Couette and Poiseuille velocity flow profiles and the Poiseuille mass flow rate (all in planar-wall channel geometries). 


\section{$2 \quad$ Velocity slip}

The commonly-used velocity slip boundary condition proposed by Maxwell [5] has the following form for isothermal cases:

$$
\boldsymbol{U}_{\text {slip }}=-\frac{2-\sigma}{\sigma} \frac{\lambda}{\mu} \boldsymbol{\tau}_{\text {wall }}
$$

where $\boldsymbol{U}$ is the mass average velocity vector, $\boldsymbol{\tau}$ is the viscous stress vector ${ }^{1}$, tangential to the surface, and $\mu$ is the gas dynamic viscosity. The tangential momentum accommodation coefficient, $\sigma$, describes the proportion of molecules being reflected diffusively $(\sigma=1)$ from the wall as opposed to those that experience specular reflections $(\sigma=0)$. If the reflections of the molecules are diffusive their tangential momentum is, on average, lost relative to the wall, as opposed to specular reflections where the tangential momentum is retained. In Eq. (2), "slip" denotes the velocity difference between the wall and the gas next to the wall, for which the notation "wall" is used.

We obtain a first order velocity slip by inserting the tangential viscous stress vector of the NS equations into Eq. (2). The velocity slip for planar walls can then be expressed as:

$$
\boldsymbol{U}_{\text {slip }}=-\frac{2-\sigma}{\sigma} \lambda\left(\frac{\partial \boldsymbol{U}}{\partial y}\right)_{\text {wall }}=-C_{1} \lambda\left(\frac{\partial \boldsymbol{U}}{\partial y}\right)_{\text {wall }}
$$

where $y$ is the coordinate perpendicular to the wall and in this case $y=0$ is the middle of the channel. ${ }^{2}$ As listed by Karniadakis et al. [7], while the coefficient $C_{1}$ is set to 1 by many investigators, Cercignani [7] uses $C_{1}=1.1466$. Following the latter, we set the coefficient

\footnotetext{
${ }^{1}$ The viscous tangential stress vector, $\boldsymbol{\tau}$, relates to the viscous stress tensor, $\boldsymbol{\Pi}$, through the expression $\boldsymbol{\tau}=(\hat{n} \cdot \boldsymbol{\Pi}) \cdot(I-\hat{n} \hat{n})$, where $\hat{n}$ is the unit vector normal to a surface and $I$ is the identity tensor [6].

${ }^{2}$ In Eq. (3) and Eq. (4) the "-" sign is applied to the first order velocity gradient if the boundary is in the direction of increasing $y$, otherwise "-" is replaced with "+" in these equations.
} 
$C_{1}=1.1466$ when using the conventional NS with first order slip.

Some investigators of micro-gas-flows argue that second order velocity slip boundary conditions should be used when modelling gas flows in the transition regime $[3,7,8]$. There are several suggested formulations for second order velocity slip; for planar walls and for our coordinate system most have the following form:

$$
\boldsymbol{U}_{\text {slip }}=-C_{1} \lambda\left(\frac{\partial \boldsymbol{U}}{\partial y}\right)_{\text {wall }}-C_{2} \lambda^{2}\left(\frac{\partial^{2} \boldsymbol{U}}{\partial y^{2}}\right)_{\text {wall }},
$$

and there are various proposals for the modelling parameters $C_{1}$ and $C_{2}$. Some are purely theoretically derived, whereas others have been obtained through comparisons with experimental results [8]. The commonly used value for $C_{1}$ in the second order slip is the same as for the first order slip, and the $C_{2}$ parameter varies in a wide range from -0.5 to $5 \pi / 12$. We choose to use, for NS using second order slip, Cercignani's proposed slip boundary coefficients $C_{1}=1.1466$ and $C_{2}=0.647[7]$.

Generally, kinetic derivations of the velocity slip such as the one performed by Cercignani are based on a diffuse reflection approximation for the gas/surface interaction [9]. An alternative approach to this is to use a correction for the gas/surface interaction using the coefficient $(2-\sigma) / \sigma$ in Eq. (2). We are interested in the way the surface not only modifies the slip coefficients but also affects the mean free path locally. Therefore, we start with the formal first and second order velocity-slip expressions, Eqs. (3) and (4), respectively, and use instead a mean free path modified to incorporate the effect of a surface, $\lambda_{\text {eff }}$, i.e.

$$
\boldsymbol{U}_{\text {slip }}=-A_{1} \lambda_{\text {eff }}\left(\frac{\partial \boldsymbol{U}}{\partial y}\right)_{\text {wall }}
$$

and

$$
\boldsymbol{U}_{\text {slip }}=-A_{2} \lambda_{\text {eff }}\left(\frac{\partial \boldsymbol{U}}{\partial y}\right)_{\text {wall }}-A_{3} \lambda_{\text {eff }}^{2}\left(\frac{\partial^{2} \boldsymbol{U}}{\partial y^{2}}\right)_{\text {wall }}
$$


These expressions have coefficients $A_{1}, A_{2}$ and $A_{3}$ different from the conventional coefficients using $C_{1}$ and $C_{2}$. This is because $\lambda_{\text {eff }}$ is expected to incorporate some surface effects, most likely requiring a change in the slip coefficient values. The conventional velocity-slip definitions represent surface effects through their slip coefficients rather than through the mean free path model.

In what follows, we present a model for the mean free path that takes into account the bounding solid surfaces.

\section{Navier-Stokes equations and geometry-dependent vis-}

\section{cosity}

Since micro-gas-flows have a large ratio of their confining boundary areas to their volumes, in the fluid mechanical model we should account for gas molecular collisions with the solid boundaries in addition to accounting for intermolecular collisions. This modelling modification is expected to have a significant effect only for confined micro-gas-flows, with negligible effect for larger scale gas-flows.

Here we use the relationship:

$$
\mu=\rho \frac{\lambda}{\sqrt{\pi / 2 R T}}
$$

which is discussed in further depth by Cercignani [9], relating the dynamic viscosity, $\mu$, to the mean free path, $\lambda$, with $\rho$ the gas density, $R$ the specific gas constant and $T$ the gas temperature. We assume that Eq. (7), which is normally valid only for gases in local equilibrium far from surfaces, remains formally valid at the surface even after taking into 
account that the mean free path is affected by gas molecular collisions with surfaces. If the "unconfined" expression for the mean free path, $\lambda$, is replaced by an "effective" and geometry-dependent mean free path, $\lambda_{\text {eff }}$, we obtain a non-constant, geometry-dependent, "effective" viscosity, $\mu_{\text {eff }}$, that can be used in the momentum conservation equation:

$$
\boldsymbol{\Pi}=\mu_{\mathrm{eff}}\left[\nabla \boldsymbol{U}+(\nabla \boldsymbol{U})^{t}\right]+\left(\frac{2}{3} \mu_{\mathrm{eff}}-\kappa\right)(\nabla \cdot \boldsymbol{U}) I
$$

where $\kappa$ is the bulk viscosity, $I$ is the identity tensor and $t$ is the transpose operator. Our modification in Eq. (8) is analogous to replacing the NS stress expression with the Burnett (or other high-order) stress expression in the general momentum conservation equation.

For many steady state micro-gas-flow situations the flow is extremely slow. This means that the inertia term, $\rho(\boldsymbol{U} \cdot \nabla) \boldsymbol{U}$, can be discarded. The conventional NS equations then reduce to the Stokes equation [7], which for our new approach would have the following form:

$$
\nabla \cdot \mu_{\text {eff }}\left[\nabla \boldsymbol{U}+(\nabla \boldsymbol{U})^{t}\right]=\nabla p
$$

We now need to derive a new expression for the mean free path, $\lambda_{\text {eff }}$, and hence obtain $\mu_{\text {eff }}$.

\section{Previous effective mean free path models}

The idea of using transport parameters that are influenced by an effective mean free path can be traced back to Stops [10]. Stops investigates the probability density

$$
p(r)=\frac{1}{\lambda} \exp \left(-\frac{r}{\lambda}\right)
$$

describing the distribution of the molecular free path in terms of the free flight length $r$. The value of the unconfined, conventional mean free path, when no solid boundaries are present, can then be obtained by integrating $r p(r)$, with respect to $r$, from zero to infinity. 
The effective mean free path expression developed by Stops, $\lambda_{\text {eff(S) }}$, is derived by using solid-angle-analysis and by shortening the upper integrational limit of $r$ from infinity to the distance to the confining wall. The integration of $r p(r)$ then yield $\lambda_{\text {eff(S) }}=\lambda K_{(S)}(y, \lambda, H)$ for molecules in a planar wall confinement, where $H$ is the wall spacing and

$$
\begin{aligned}
K_{(S)}(y, \lambda, H)= & \frac{1}{2}\left\{2+\left(\frac{H / 2+y}{\lambda}-1\right) \exp \left(-\frac{H / 2+y}{\lambda}\right)-\left(\frac{H / 2+y}{\lambda}\right)^{2} \operatorname{Ei}\left(\frac{H / 2+y}{\lambda}\right)\right. \\
& \left.+\left(\frac{H / 2-y}{\lambda}-1\right) \exp \left(\frac{H / 2-y}{\lambda}\right)-\left(\frac{H / 2-y}{\lambda}\right)^{2} \operatorname{Ei}\left(\frac{H / 2-y}{\lambda}\right)\right\} .
\end{aligned}
$$

This expression is used by Guo et al. [4] in solving their micro-gas-flows. The function Ei in Eq. (11) is the exponential integral function defined as:

$$
E i(z)=\int_{1}^{\infty} t^{-1} \exp (-z t) d t
$$

In the next section we derive a model similar to $\lambda_{\text {eff(S) }}$ but without the dependence on the Ei(z)-function, which may therefore be easier to implement and more computationally efficient for micro-gas-flow calculations.

\section{A probability function-based effective mean free path}

\section{model}

We use instead the integrated form of the density function $p(r)$, defined in Eq. (10), referred to as the probability function, i.e.

$$
P(r)=\int p(r) d r=C-\exp \left(-\frac{r}{\lambda}\right)
$$


This function describes the probability a molecule travels a distance $r+d r$ without experiencing a collision. The integration constant, $C$, is set to one so that the probability ranges from zero to one.

Our model is derived for the two-planar-wall configuration shown in Fig. 1. We use the notation $r^{-}$if the molecule is travelling in the negative $y$-direction, and $r^{+}$if the molecule is travelling in the positive $y$-direction. We also use the notations $\theta^{-}$and $\theta^{+}$for the equally probable zenith angle travelling direction of the molecule. These quantities are related through $r^{-}=(H / 2+y) / \cos \left(\theta^{-}\right)$and $r^{+}=(H / 2-y) / \cos \left(\theta^{+}\right)$.

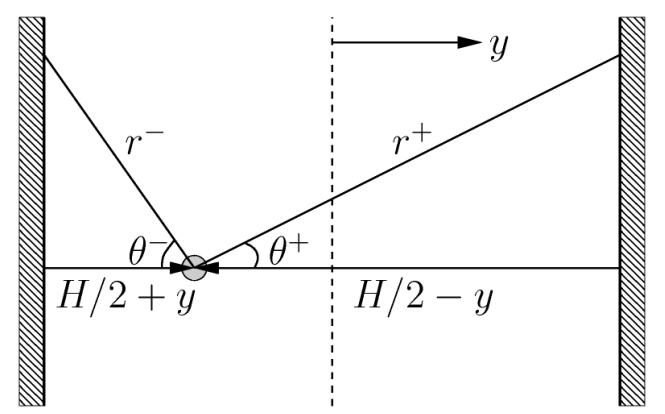

Figure 1: A molecule confined between two planar walls with spacing $H$. The molecule has an equal probability to travel in any zenith angle $\theta^{-}$or $\theta^{+}$or to travel in either the positive or negative $y$-direction. The molecule under consideration is assumed to have just experienced an intermolecular collision at its current position $H / 2-y$.

The molecular free path, $l$, is retrieved by weighting the unconfined molecular mean free path, $\lambda$, with $P$ as follows:

$$
\begin{aligned}
l & =\lambda \frac{1}{2}\left[P\left(r^{-}\right)+P\left(r^{+}\right)\right] \\
& =\lambda\left\{1-\frac{1}{2}\left[\exp \left(-\frac{r^{-}}{\lambda}\right)+\exp \left(-\frac{r^{+}}{\lambda}\right)\right]\right\} .
\end{aligned}
$$

A 3-dimensional mean free path depending on the molecule's distance to a wall is then HT-08-1426 
obtained by averaging the free path with respect to $\theta^{-}$and $\theta^{+}$in the range $[0, \pi / 2]$ using the mean integral theorem,

$$
\left\langle X\left(\theta^{*}\right)\right\rangle=\frac{2}{\pi} \int_{0}^{\pi / 2} X\left(\theta^{*}\right) d \theta^{*}
$$

where the integrational domain is illustrated in Fig. 2 for a molecule travelling in the negative $y$-direction. Averaging over the free path in Eq. (14) may be done using Simpson's numerical

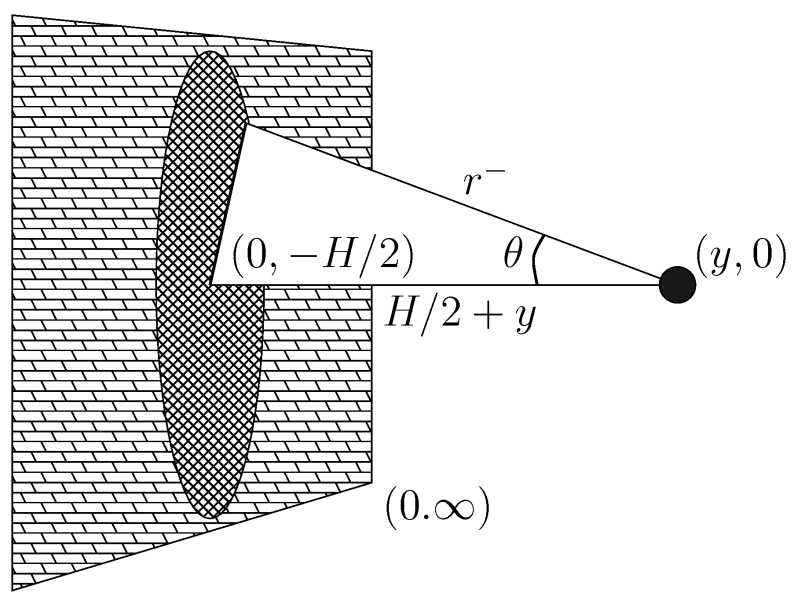

Figure 2: A molecule at a distance $H / 2+y$ from a planar wall; possible trajectories for a molecule travelling in the negative $y$-direction in cylindrical coordinates $[H / 2+y,(H / 2+$ $y) \times \tan \theta]$, where $\infty$ denotes infinity. 
integration involving 14 subintervals $^{3}$ results in $\lambda_{\text {eff }}=\lambda K(y, \lambda, H)$, where

$$
\begin{aligned}
K(y, \lambda, H)= & -\frac{1}{82}\left[\exp \left(-\frac{H / 2+y}{\lambda}\right)+\exp \left(-\frac{H / 2-y}{\lambda}\right)\right. \\
& +4 \sum_{i=1}^{7} \exp \left(-\frac{H / 2+y}{\lambda \cos [(2 i-1) \pi / 28]}\right) \\
& +4 \sum_{i=1}^{7} \exp \left(-\frac{H / 2-y}{\lambda \cos [(2 i-1) \pi / 28]}\right) \\
& \left.+2 \sum_{i=1}^{6} \exp \left(-\frac{H / 2+y}{\lambda \cos [\pi i / 14]}\right)+2 \sum_{i=1}^{6} \exp \left(-\frac{H / 2-y}{\lambda \cos [\pi i / 14]}\right)\right] .
\end{aligned}
$$

In the remainder of this paper we will focus on the $y$-dependence of $K$, as $\lambda$ and $H$ are determined through the rarefaction parameter $K n$ and the micro-channel geometry, respectively. The different $K$-functions, Eq. (11) and Eq. (16), are compared in Fig. 3 for four $K n: K n_{A}=0.04, K n_{B}=0.25, K n_{C}=1$ and $K n_{D}=20$. It is seen that both models show similar results for all $K n$-cases, with the largest difference at $K n_{C}$. By inspection of the $K n_{A}$-case, both models fulfil the physically intuitive requirements that

$$
K(0) \approx 1 \text { and } K(H / 2) \approx \frac{1}{2}
$$

The requirement at the wall can be realised by considering the average of the equal probabilities of a molecule travelling in the direction towards the confining wall (not yielding any travelling length contribution) and the probability of it travelling into the bulk of the flow (yielding a contribution of the length $\lambda$ ). The requirement for molecules far away from the wall is that the effective mean free path should approach its conventional unconfined value. For $K n_{B}=0.25$, the channel is four unconfined mean free paths wide; since the

\footnotetext{
${ }^{3}$ The difference in our mass flow results, see below, for 14 and 16 integration intervals is $1.54 \%$ for $K n=1$, indicating that further increase of the number of integration intervals will only marginally affect the results.
} 


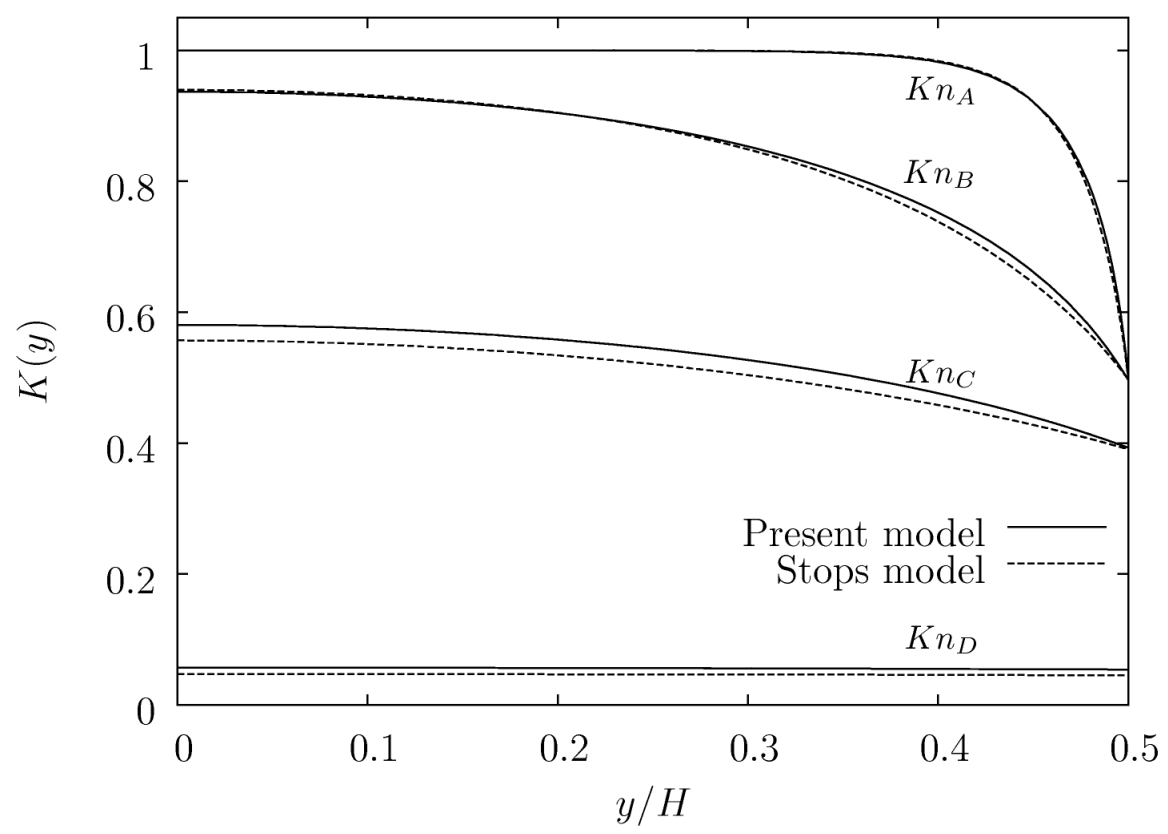

Figure 3: Comparison of different $\lambda_{\text {eff- }}$ models in a half-channel for different Knudsen numbers, where $\lambda_{\text {eff }}=\lambda K(y)$ and $K n_{A}=0.04, K n_{B}=0.25, K n_{C}=1$ and $K n_{D}=20$.

effective mean free path almost achieves the conventional unconfined value at $y=0$, the Knudsen-layer can be said to be approximately two unconfined mean free paths wide.

For higher $K n$, the entire $K(y)$ profile is lowered due to Knudsen-layer overlap and thereby an increasing likelihood of wall collisions. The basic physical requirement of decreasing $K(y)$ with increasing $K n$ can be seen by inspection of the considerably lower profile of the $K n_{D}$-case compared to the other cases. The $K n_{B}$ and $K n_{C}$ cases represent intermediate states between the $K n_{A}$ and $K n_{D}$ cases, where the profile near the wall is lower than the near-wall requirement in Eq. (17) because a molecule close to one of the walls has a significant probability of travelling directly to the other side of the channel and colliding with that wall, which results in a contribution of less than a mean free path for this travelling direction. 
In the next section we present simple flow test cases using our $\lambda_{\text {eff-dependent parameter }}$ $\mu_{\text {eff }}$ within the Navier-Stokes equations.

\section{Test case results}

Isothermal, fully-developed velocity profiles in planar Couette and Poiseuille flow are calculated from our modified Stokes equation:

$$
\frac{\partial}{\partial y}\left[\mu_{\mathrm{eff}} \frac{\partial U_{x}}{\partial y}\right]=\frac{\partial p}{\partial x},
$$

which uses an effective viscosity derived by using $\lambda_{\text {eff }}$ in Eq. 7 . The velocity $U_{x}$ is in the axial direction ( $x$-direction) of the channel and it is assumed to vary only in the direction normal to the wall, the $y$-direction. This model is applied in turn with the first order velocity slip of Eq. (5) and then with the second order velocity slip of Eq. (6), in which $\lambda$ is again replaced by its near-wall value, at $y=H / 2$, of $\lambda_{\text {eff }}$. As this is now a different slip model than the conventional one, we choose to set the coefficients $A_{1}=1$ for our first order velocity slip and $A_{2}=0.05$ and $A_{3}=0.63$ for our second order velocity slip; These values for our present model are chosen purely based on which produce the best velocity profile results in the Couette and Poiseuille flows, and the mass flow rate in the Poiseuille flow. Since our present model uses a slip definition that has a different velocity gradient at the wall compared to NS, and we apply a wall value of $\lambda_{\text {eff }}$ instead of the unconfined value, it is not expected that the slip coefficients should have their conventional values.

It is shown by experimental investigators, like Turner et al. [11], that micro-gas-flows have compressible characteristics even when the flow velocities are less than Mach 0.3. Due to this, we use the same solution method to solve for this compressible flow as in Kandlikar et 
al. [12], i.e. the normalised velocity profile in the channel cross section of the flow is obtained by assuming fully-developed incompressible flow, but the density is then recalculated using the ideal gas law. The mass flow rate in the Poiseuille flow case is calculated through cross-channel integration of the velocity profiles.

\subsection{Couette flow}

In Couette flow the pressure gradient of Eq. (18) is assumed negligible, yielding the governing equation:

$$
\frac{\partial}{\partial y}\left(\mu_{\mathrm{eff}} \frac{\partial U_{x}}{\partial y}\right)=0
$$

where $\mu_{\mathrm{eff}}=\mu K(y, \lambda, H)$. The solution to this equation, which we refer to as the "NS $\mathrm{eff}^{-}$ solution", using the first order $\lambda_{\text {eff-dependent velocity slip, is }}$

$$
\frac{U_{x}}{U_{w}}=\frac{F(y)-F(y=0)}{F(y=H / 2)+A_{1} \lambda-F(y=0)}
$$

where

$$
F(y)=\int \frac{1}{K(y)} d y
$$

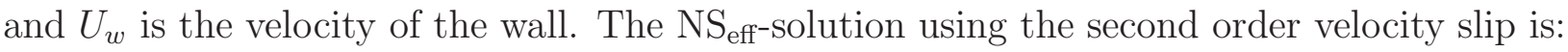

$$
\frac{U_{x}}{U_{w}}=\frac{F(y)-F(y=0)}{F(y=H / 2)+A_{2} \lambda-A_{3} \lambda^{2} K^{\prime}(y=H / 2)-F(y=0)},
$$

where

$$
K^{\prime}(y)=\frac{d K(y)}{d y}
$$

The conventional solution to Eq. (19), with constant viscosity and boundary conditions 
applied using the conventional $\lambda$, is as follows ${ }^{4}$ :

$$
\frac{U_{x}}{U_{w}}=\frac{y}{H / 2+C_{1} \lambda}
$$

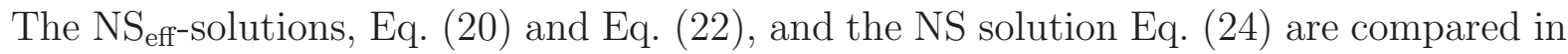
Figs. 4 and 5 for five different $K n$ of $0.01,0.04,0.08,0.113$ and 0.339 , alongside the kinetic BGK-model solution obtained by Sharipov [13].

Figure 4 shows that for $K n_{E}=0.01$ all of the fluid model solutions have a sufficiently linear profile to match the BGK-result; the $\mathrm{NS}_{\text {eff }}$ model with second order velocity slip best captures the amount of slip. For the $K n_{B}$ and the $K n_{F}$ cases, the non-linear velocity profile at the wall is evident and is captured by both our $\mathrm{NS}_{\text {eff }}$ models. The bulk of the flow is also considered to be well described by our models, although there is a slight deviation for all models for the $K n_{F}$-case.

Figure 5 shows the $K n_{E}$-case again for reference. In the $K n_{G}$-case the slip amount and the near wall curvature is captured by our models, while the conventional NS model captures the bulk velocity profile best, in comparison to the BGK-results. In the $K n_{H^{-}}$ case the conventional NS model deviates quite severely from the BGK-results through the channel; our first order slip model captures the amount of slip best, while our second order slip model captures the bulk velocity profile the best.

\footnotetext{
${ }^{4}$ The second order velocity slip solution is the same as the first order slip solution, because the second gradient of the velocity does not exist in this test case.
} 


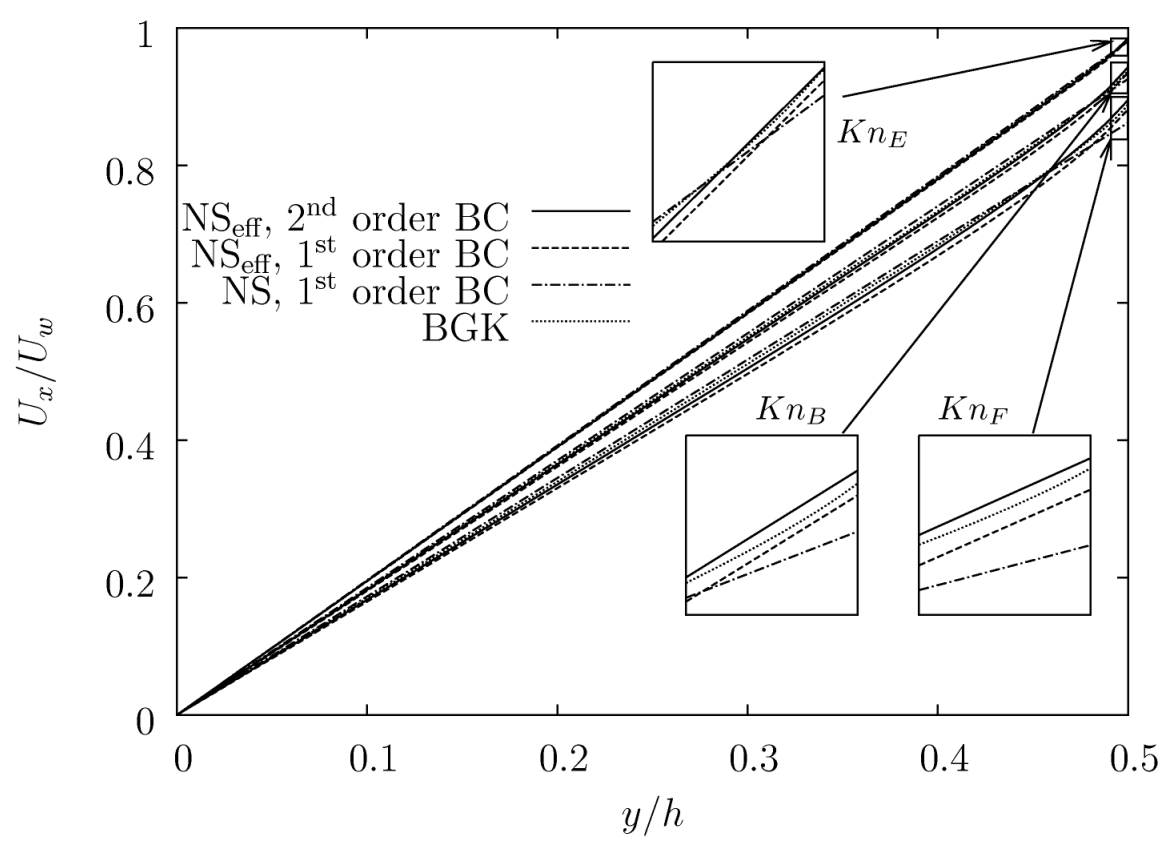

Figure 4: Half-channel Couette flow velocity profiles using conventional Navier-Stokes (NS) and our effective viscosity model ( $\mathrm{NS}_{\text {eff }}$ ), using first and second order boundary conditions (BC), compared with the BGK results of Sharipov [13]. The velocity profiles are for $K n_{E}=0.01, K n_{\mathrm{B}}=0.04$ and $K n_{F}=0.08$, and $y=0$ is the channel centre. The slip coefficients for our second order model are $A_{2}=0.05$ and $A_{3}=0.63$, and for our first order model $A_{1}=1$.

\subsection{Poiseuille flow}

We now calculate the velocity profiles and the mass flow rate for isothermal, fully-developed Poiseuille flow in a planar-wall channel. 


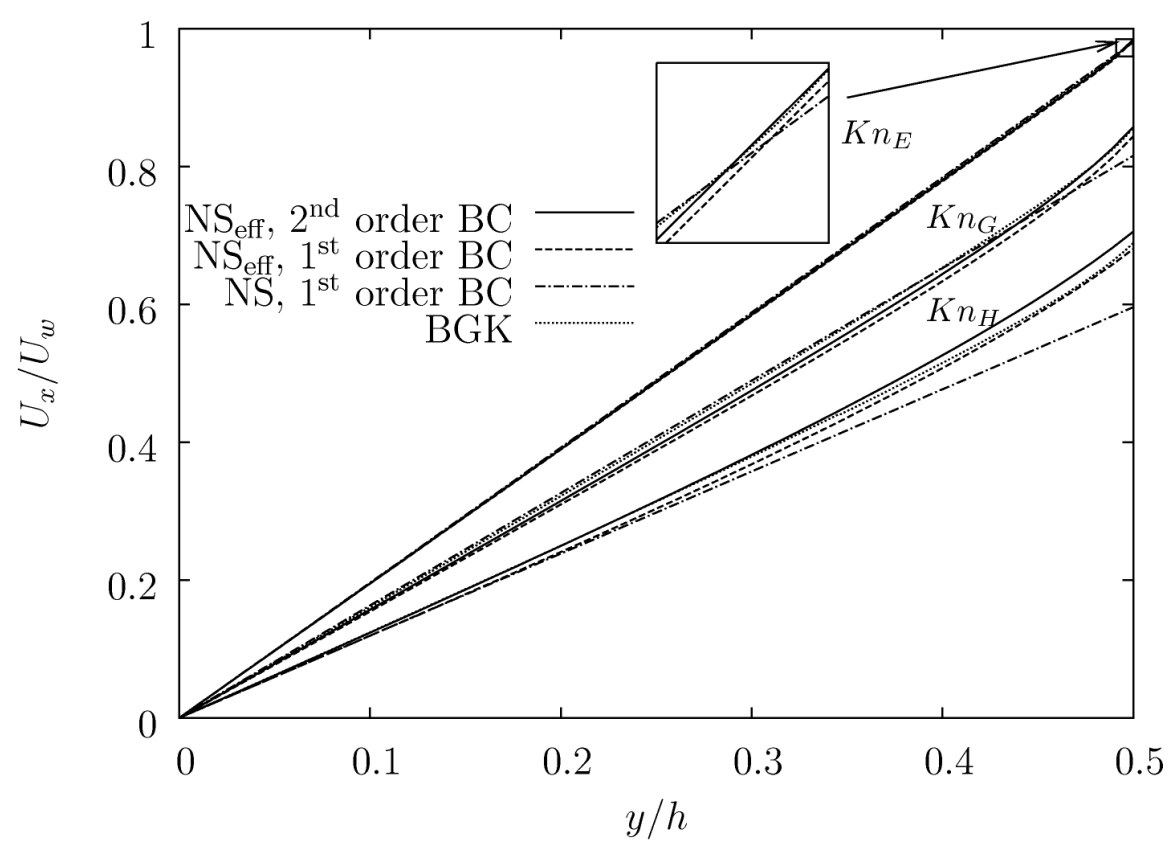

Figure 5: Couette flow velocity profiles using conventional Navier-Stokes (NS), and our effective viscosity model ( $\left.\mathrm{NS}_{\text {eff }}\right)$, with first and second order boundary conditions (BC), compared with the BGK results of Sharipov [13]; $K n_{E}=0.01, K n_{\mathrm{G}}=0.113$ and $K n_{H}=0.339$, and $y=0$ is the channel centre. The coefficients for our second order slip model are $A_{2}=0.05$ and $A_{3}=0.63$, and for our first order model $A_{1}=1$.

\subsubsection{Velocity profile results}

In this case the solution to Eq. (18) using our $\mathrm{NS}_{\text {eff }}$ model with first-order $\lambda_{\text {eff-dependent }}$ velocity slip is as follows:

$$
\frac{U_{x}}{U_{0}}=\frac{8}{H^{2}}\left[G(H / 2)+A_{1} \lambda \frac{H}{2}-G(y)\right]
$$

where

$$
G(y)=\int \frac{y}{K(y)} d y \quad \text { and } \quad U_{0}=-\frac{H^{2}}{8 \mu} \frac{d p}{d x} .
$$


The $\mathrm{NS}_{\text {eff-Solution with second-order }} \lambda_{\text {eff-dependent velocity slip is: }}$

$$
\frac{U_{x}}{U_{0}}=\frac{8}{H^{2}}\left[G(H / 2)+A_{2} \lambda \frac{H}{2}+A_{3} \lambda^{2}\left[K(H / 2)-\frac{H}{2} K^{\prime}(H / 2)\right]-G(y)\right]
$$

where

$$
K^{\prime}(y)=\frac{d K(y)}{d y}
$$

The solution of the conventional NS equation, with constant viscosity and first-order velocity slip using the unconfined $\lambda$, is:

$$
\frac{U_{x}}{U_{0}}=1-4\left(\frac{y}{H}\right)^{2}+4 C_{1} \frac{\lambda}{H}
$$

and the NS-solution with second-order velocity slip using the unconfined $\lambda$ is:

$$
\frac{U_{x}}{U_{0}}=1-4\left(\frac{y}{H}\right)^{2}+4 C_{1} \frac{\lambda}{H}+8 C_{2}\left(\frac{\lambda}{H}\right)^{2} .
$$

In Figs. 6 and 7 the results of these various NS-based models are shown together with results from the BGK model presented by Sharipov [13], for various $K n$ as listed in the captions.

In Fig. 6 it is seen for all $K n$-cases that both our present models describe the bulk velocity profiles and the near wall region quite well. For the $K n_{B}$ and $K n_{F}$ cases the conventional NS equations with second order slip describe the bulk velocity profile well but do not capture the same curvature as the BGK-model at the wall. The conventional NS with first order slip deviates significantly in the $K n_{B}$ case and even more in the $K n_{F}$, case as expected.

In Fig. 7 in the $K n_{G}$ case it is seen that only our two present models manage to match the velocity profile of the BGK-model. In the $K n_{H}$ case it is only our present model using second order slip that captures the same slip amount as the BGK-model. While none of the 


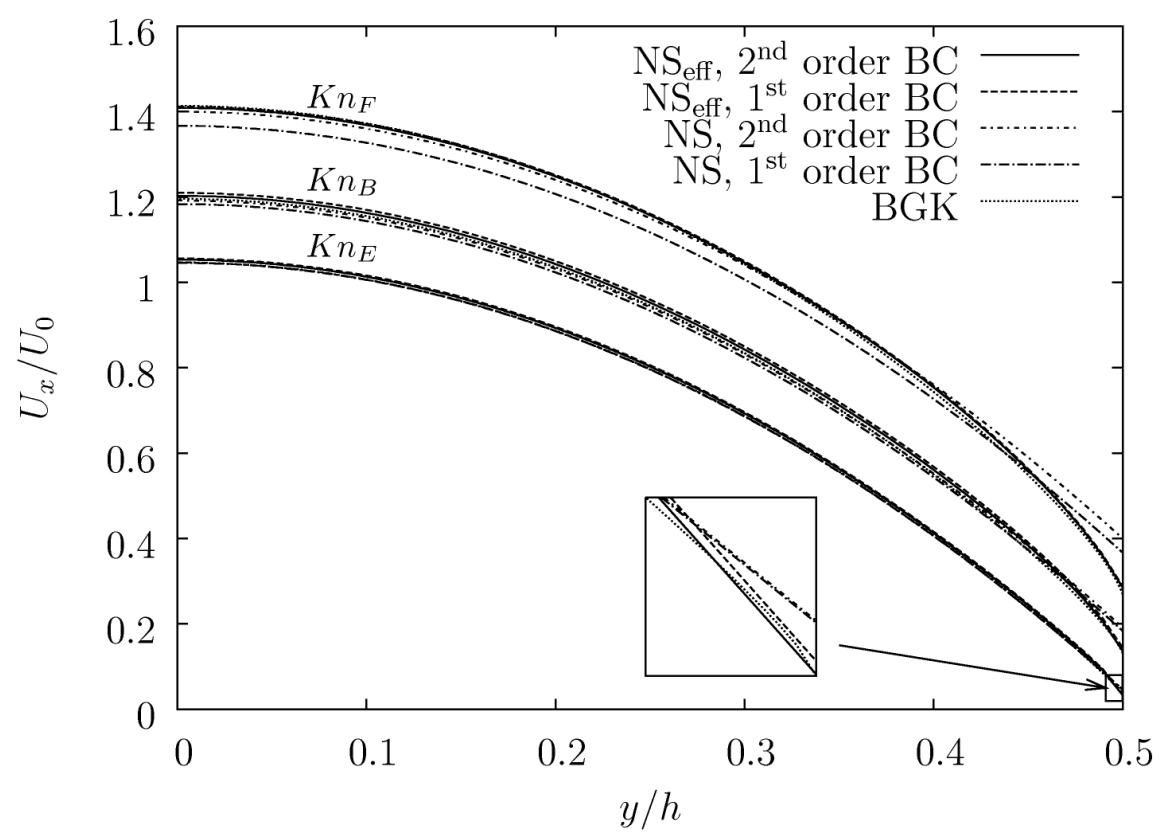

Figure 6: Half-channel Poiseuille flow velocity profiles using conventional NS and our effective viscosity model $\mathrm{NS}_{\text {eff }}$, using first and second order boundary conditions (BC), compared with the BGK results of Sharipov [13]. The velocity profiles are for $K n_{E}=0.01, K n_{B}=0.04$ and $K n_{F}=0.08$, and $y=0$ is the channel centre. The slip coefficients for our second order model are $A_{2}=0.05$ and $A_{3}=0.63$, and for our first order model $A_{1}=1$.

NS-based models capture the same velocity profile as the BGK-model, our present models have a higher amplitude of the profile relative to the conventional NS-models, which suggests that our present models are in closer agreement with the BGK model for the $K n_{H}$-case. In the $K n_{I}$-case only the results of our present model with second order slip are compared to the BGK-model because the other models deviate to a too great extent. As can be seen, our model captures a reasonable average velocity across the channel, although we still cannot capture flow predictions correctly in the high Knudsen number range from about 0.903 . 


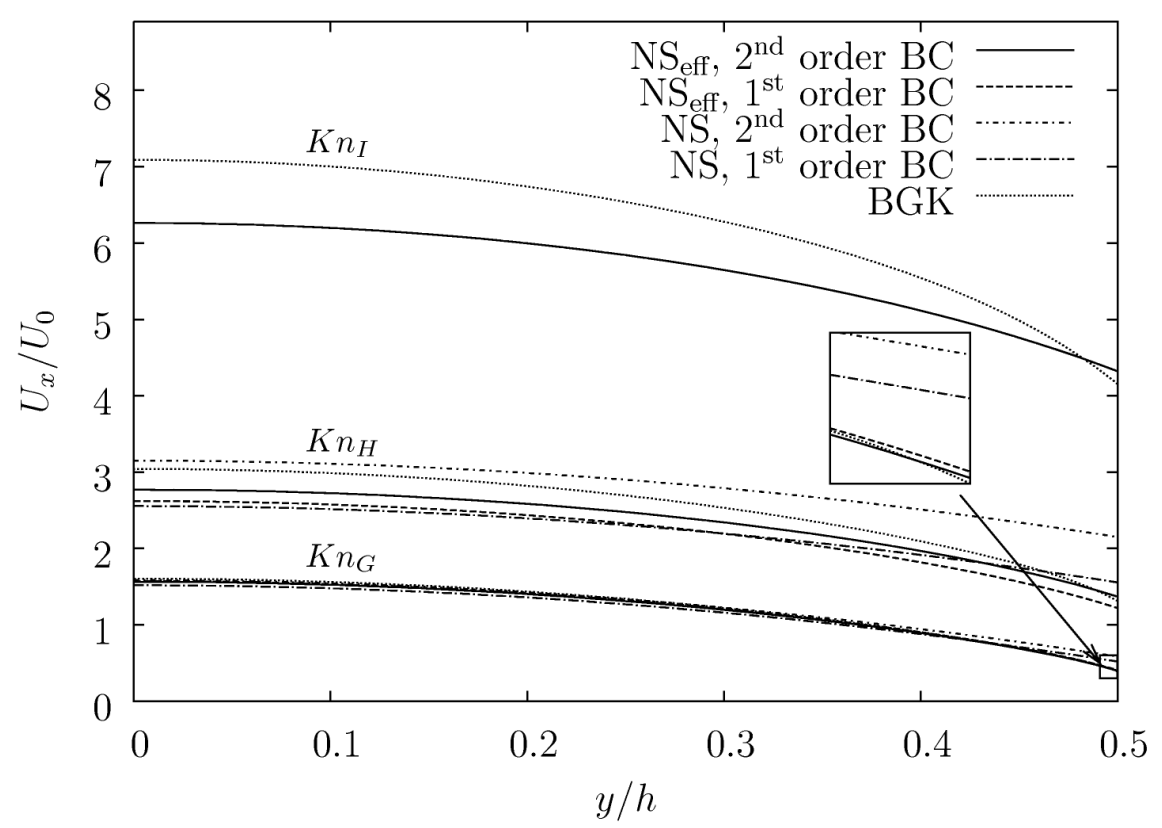

Figure 7: Half-channel Poiseuille flow velocity profiles using conventional NS, and our effective viscosity model $\mathrm{NS}_{\text {eff }}$, with first and second order boundary conditions (BC), compared with the BGK results of Sharipov [13]; $K n_{G}=0.113, K n_{H}=0.339$ and $K n_{I}=0.903$, and $y=0$ is the channel centre. The coefficients for our second order slip model are $A_{2}=0.05$ and $A_{3}=0.63$, and for our first order model $A_{1}=1$.

\subsubsection{Mass flow results}

We now compare the mass flow rates predicted by the present models with experimental results by Ewart et al. [8] for various degrees of rarefaction. Ewart's experimental measurements are made for helium gas, driven by a pressure ratio of 5 between the inlet and the outlet of the channel. Mass flow rates are obtained in the $K n$-range of 0.03 to 50 . The experimental channel dimensions are: height, $H=9.38 \mu m$; width, $W=492 \mu m$; and length, $L=9.39 \mathrm{~mm}$. Since this channel is wide compared to its height it is assumed that a com- 
parison with our model for just two planar walls is valid. However, according to Sharipov [13] there is still an influence of the lateral walls, here separated by $W$; the error due to this influence can be taken into account by multiplying the mass predicted flow rate by $1-0.63 H / W=0.99$.

In order to compare our results with experiment, the velocity-dependent mass flow rate is calculated using the following relation:

$$
\dot{m}=\rho\left\langle U_{x}\right\rangle A=\frac{p\left\langle U_{x}\right\rangle A}{R T}
$$

where $A$ is the area of the cross section of the channel and

$$
\widetilde{U}_{x}=\frac{\left\langle U_{x}\right\rangle}{U_{0}}=\frac{2}{H} \int_{0}^{H / 2} \frac{U_{x}}{U_{0}} d y
$$

is the normalised average velocity across the channel width. We will here use the mass flow definition of Eq. (31) with the averaged velocities of the four NS-based velocity expressions given in Eqs. $(29,30,25,27)$ and normalise by the quantity

$$
\dot{m}_{0}=-\frac{A H}{\sqrt{2 R T}} \frac{d p}{d x}
$$

We then obtain the normalised expression for the mass flow rate,

$$
\frac{\dot{m}}{\dot{m}_{0}}=\frac{U_{0}}{\dot{m}_{0}} \frac{p A}{R T} \widetilde{U}_{x}=\frac{\sqrt{\pi}}{8} \frac{H}{\lambda} \widetilde{U}_{x}=\frac{\delta}{4} \widetilde{U}_{x}(\delta)
$$

where the inverse rarefaction parameter is

$$
\delta=\frac{\sqrt{\pi}}{2} \frac{H}{\lambda} .
$$

The results of the mass flow rates of the NS and $\mathrm{NS}_{\text {eff }}$ models are shown in Fig. 8, together with the BGK results reported by Sharipov [13] and the experimental results of Ewart et 
al. [8]. In Table 1 we also list an approximate range of applicability, within the $\delta$-inspection range of $0.3-20$, for the NS-based models and the BGK-model. These applicability ranges are estimated by the ability of the different models to reproduce mass flow rates similar to the experimental data within a reasonable range of the stated error bars.

Figure 8 shows that all of the models are asymptotic to the experimental data for low $K n$ (i.e. high $\delta$ ), which strengthens our presumption that the influence of the effective viscosity and the effective mean free path should decay with decreasing degree of rarefaction. Our

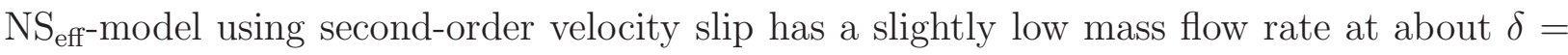
2, compared with the validation data of the BGK-method [13] and experimental data [8]. This result can also be seen by inspection of the velocity profile for the $K n_{I^{-}}$case of Fig. 7 , where the velocity profile is low in comparison to the BGK-model. In Fig. 8 it is only the second order slip models of the NS-based models that capture the mass flow minimum, which occurs for the conventional model at about $\delta=2$ and for the present model at $\delta=1$. The comparison data of the BGK-model and the experimental data have a minimum at $\delta=1.2$. The conventional NS-model with second order slip has a mass flow rate that is unbounded and therefore unphysical for low $\delta$-values.

\section{Discussion and conclusions}

A velocity slip boundary condition is required in the application of the continuum NavierStokes equations to micro-gas-flows in the slip regime in order to achieve better predictions. In this paper we have presented a continuum fluid model for micro-gas-flows in the lower transition-continuum regime by incorporating a molecular collision length description into 


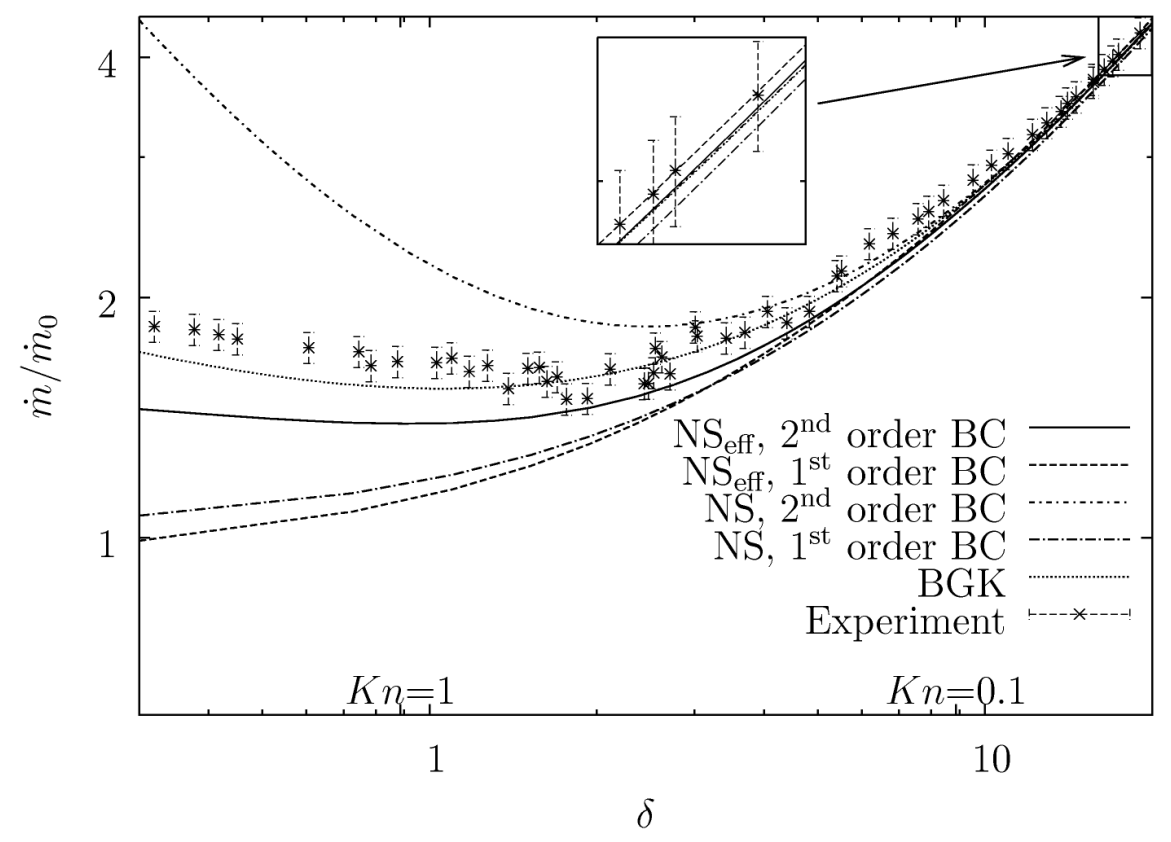

Figure 8: Mass flow results from conventional NS and our effective viscosity model $\mathrm{NS}_{\text {eff }}$, using first and second order boundary conditions (BC). The results are compared with BGK solutions by Sharipov [13] and experimental results by Ewart et al. [8]. The height of the error bars of the experimental data is set to $4.5 \%$ of the normalised mass flow rate values, consistent with the data in [8]. The coefficients for our second order slip model are $A_{2}=0.05$ and $A_{3}=0.63$, and for our first order slip model $A_{1}=1$.

the Navier-Stokes equations, as well as using first- and second-order velocity slip boundary conditions. Our molecular description takes into account gas molecular collisions with boundaries in the conventional definition of the mean free path, which therefore becomes a geometry-dependent and "effective" parameter varying with distance from a solid surface. This new definition of the effective mean free path yields an effective viscosity, and hence a non-linear stress/strain-rate constitutive relationship in the Navier-Stokes framework. The 
Table 1: Applicability ranges of the tested NS-based models, estimated by comparing the model predictions of mass flow rates with the experimental data of Ewart et al. [8].

\begin{tabular}{|l|c|c|}
\hline \multirow{2}{*}{ Model } & \multicolumn{2}{|c|}{ Approximate range of applicability } \\
& In terms of $\delta$ & In terms of $K n$ \\
\hline $\mathrm{NS}_{\text {eff }}$, second order BC & $2-20$ & $0.04-0.44$ \\
\hline $\mathrm{NS}_{\text {eff }}$, first order BC & $4-20$ & $0.04-0.22$ \\
\hline $\mathrm{NS}$, second order BC & & $0.04-0.30$ \\
\hline $\mathrm{NS}$, first order BC & $3-20$ & $0.04-0.06$ \\
\hline BGK & $4-15$ & $0.04-2.95$ \\
\hline
\end{tabular}

${ }^{a}$ It should be noted that the investigators Colin et al. [14] and Maurer et al. [15] found that for special cases the applicability range of NS with second order boundary condition reached up to $K n=0.25$ and $K n=0.3$ respectively.

velocity boundary conditions applied with our models then also become dependent on the mean-free-path near the wall.

Although a simple model is preferred to describe flow characteristics for a wide range of $K n$, it was found by inspection of Couette flow and Poiseuille flow results that the conventional Navier-Stokes equations are able to predict mass flows correctly up to about $K n$ $=0.30$. However, our effective mean-free-path-based model using first- and second order velocity slip showed reasonably good results for velocity profiles and mass flow up to about the same $K n$.

It should be noted that the conventional Navier-Stokes equations can produce results which fit the validation data better for higher $K n$ in the Poiseuille mass flow case. For 
example, Maurer et al. [15] present results where the conventional NS with second order velocity slip captures the mass flow correctly up to about $K n=1$, for helium and nitrogen gas using slip coefficients $C_{1}=1.2,1.3$, respectively, and $C_{3}=0.91,0.87$, respectively. In this paper we use the coefficients of Cercignani, $C_{1}=1.4466$ and $C_{2}=0.647$ [7], for the conventional Navier-Stokes equations since these coefficients present good velocity profile results to about $K n=0.01$, but above this fail to capture the near wall velocity profiles reported by the BGK-model of Sharipov [13].

For our new model we have applied coefficients that produce the best results for the velocity profiles in Couette and Poiseuille flow, as well as the mass flow rates of Poiseuille flow. For second order velocity slip, with our mean free path model, these coefficients are found to be $A_{2}=0.05$ and $A_{3}=0.63$; for our first order boundary condition the best coefficient is found to be $A_{1}=1$. Generally, our model of a modified Navier-Stokes equation with second order velocity slip produces better results than our model with only first order velocity slip. The low value of $A_{2}$ in our velocity slip formulations may be explained by the fact that our model incorporates some surface effects through the mean free path, not entirely through the slip coefficient as in Cercignani's coefficients.

The Poiseuille mass flow rate results show that only by using second-order boundary conditions can the mass flow minimum be captured. However, our model did not fully capture the velocity profile produced by the BGK-model of Sharipov [13] at $K n=0.339$, and we still cannot capture flow predictions correctly in the high Knudsen number range from about 0.903. Corrections to our description of an effective mean-free-path may, however, increase the range of applicability of our approach further into the continuum-transition regime. For example, the present description of the effective mean free path requires that 
intermolecular collisions should be accounted for in the same way as molecular collisions with the boundaries. However, intermolecular collisions cause a shortening of the free paths of both of the involved molecules, which is why we will investigate further the relationship between the unconfined mean free path and our geometry-dependent mean free path to take these differences into account. In future work we also aim to validate the geometrydependence of the molecular mean-free-path in the presence of solid-boundaries by using Molecular Dynamics simulations.

\section{Acknowledgments}

The authors would like to thank Simon Mizzi for helpful discussions and Timothée Ewart for providing experimental data, and the referees of this paper for their useful comments. This research is funded in the UK by the Engineering and Physical Sciences Research Council under grant number EP/D007488/1.

\section{References}

[1] Arkilic, E., Schmidt, M., and Breuer, K., 1997, "Gaseous slip flow in long microchannels," Journal of Microelectromechanical Systems, 6(2), pp. 167-178.

[2] Colin, S., 2005, "Rarefaction and compressibility effects on steady and transient gas flows in microchannels," Microfluidics and Nanofluidics, 1(3), pp. 268 - 279.

[3] Gad-el-Hak, M., 1999, "Fluid mechanics of microdevices - the Freeman Scholar lecture," Journal of Fluids Engineering, Transactions of the ASME, 121(1), pp. 5-33. 
[4] Guo, Z. L., Shi, B. C., and Zheng, C. G., 2007, "An extended Navier-Stokes formulation for gas flows in the Knudsen layer near a wall," Europhysics Letters, 80(2), pp. 2400124006.

[5] Maxwell, J., 1879, "On stresses in rarefied gases arising from inequalities of temperature," Philosophical Transactions of the Royal Society of London, 170, pp. 231-256.

[6] Lockerby, D. A., Reese, J. M., Emerson, D. R., and Barber, R. W., 2004, "Velocity boundary condition at solid walls in rarefied gas calculations," Physical Review E, 70(1/2), p. 017303.

[7] Karniadakis, G., Beskok, A., and Aluru, N., 2005, Microflows and Nanoflows: Fundamentals and Simulation, Springer.

[8] Ewart, T., Perrier, P., Graur, I. A., and Meolans, J. G., 2007, "Mass flow rate measurements in a microchannel, from hydrodynamic to near free molecular regimes," Journal of Fluid Mechanics, 584, pp. $337-356$.

[9] Cercignani, C., 2000, Rarefied Gas Dynamics: from Basic Concepts to Actual Calculations, Cambridge University Press.

[10] Stops, D. W., 1970, "The mean free path of gas molecules in the transition regime," Journal of Physics D, 3(5), pp. 685-696.

[11] Turner, S. E., Lam, L. C., Faghri, M., and Gregory, O. J., 2004, "Experimental investigation of gas flow in microchannels," Journal of Heat Transfer, 126(5), pp. 753-763. 
[12] Kandlikar, S., Garimella, S., Li, D., Colin, S., and King, M. R., 2005, Heat Transfer and Fluid Flow in Minichannels and Microchannels, Elsevier.

[13] Sharipov, F., 1999, "Rarefied gas flow through a long rectangular channel," Journal of Vacuum Science \& Technology A:Vacuum Surfaces and Films, 17(5), pp. 3062-3066.

[14] Colin, S., Lalonde, P., and Caen, R., 2004, "Validation of a second-order slip flow model in rectangular microchannels," Heat Transfer Engineering, 25(3), pp. 23 - 30.

[15] Maurer, J., Tabeling, P., Joseph, P., and Willaime, H., 2003, "Second-order slip laws in microchannels for helium and nitrogen," Physics of Fluids, 15(9), pp. 2613-2621. 